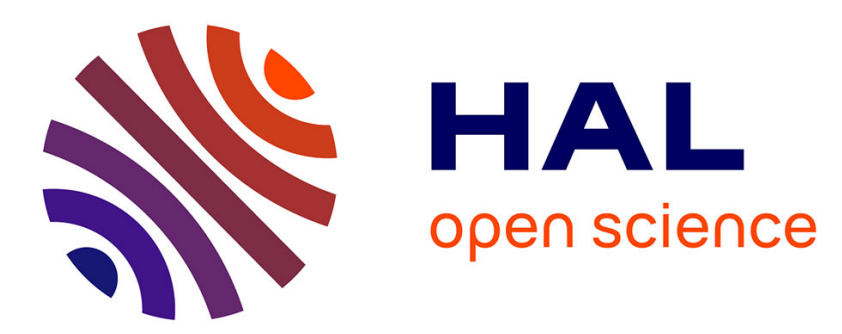

\title{
Generalized Semi-Infinite Programming: Optimality Conditions Involving Reverse Convex Problems
}

Abdelmalek Aboussoror, Samir Adly

\section{To cite this version:}

Abdelmalek Aboussoror, Samir Adly. Generalized Semi-Infinite Programming: Optimality Conditions Involving Reverse Convex Problems. Numerical Functional Analysis and Optimization, 2014, 35 (7-9), pp.816-836. 10.1080/01630563.2014.895748 . hal-01313111

\section{HAL Id: hal-01313111 \\ https://hal.science/hal-01313111}

Submitted on 16 Jul 2018

HAL is a multi-disciplinary open access archive for the deposit and dissemination of scientific research documents, whether they are published or not. The documents may come from teaching and research institutions in France or abroad, or from public or private research centers.
L'archive ouverte pluridisciplinaire HAL, est destinée au dépôt et à la diffusion de documents scientifiques de niveau recherche, publiés ou non, émanant des établissements d'enseignement et de recherche français ou étrangers, des laboratoires publics ou privés. 


\title{
GENERALIZED SEMI-INFINITE PROGRAMMING: OPTIMALITY CONDITIONS INVOLVING REVERSE CONVEX PROBLEMS
}

\author{
Abdelmalek Aboussoror ${ }^{1}$ and Samir Adly ${ }^{2}$ \\ ${ }^{1}$ Faculté Polydisciplinaire de Safi, Laboratoire LMC, Université Cadi Ayyad, Safi, Morocco \\ ${ }^{2}$ Laboratoire XLIM UMR-CNRS 6172, Département de Mathématiques, \\ Université de Limoges, Limoges, France
}

\begin{abstract}
$\square \quad$ This article deals with a generalized semi-infinite programming problem $(S)$. Under appropriate assumptions, for such a problem we give necessary and sufficient optimality conditions via reverse convex problems. In particular, a necessary and sufficient optimality condition reduces the problem $(S)$ to a min-max problem constrained with compact convex linked constraints.
\end{abstract}

Keywords Convex analysis; Generalized semi-infinite programming problems; Optimality conditions; Stability.

Mathematics Subject Classification 90C34; 90C46; 90C31; 46N10.

\section{INTRODUCTION}

We consider the following generalized semi-infinite programming problem

$$
\text { (S) } \min _{\substack{x \in X \\ G(x, y) \leq 0, \forall y \in Y(x)}} F(x)
$$

where $F: \mathbb{R}^{p} \rightarrow \mathbb{R}, G: \mathbb{R}^{p} \times \mathbb{R}^{q} \rightarrow \mathbb{R}$ are functions, $X$ is a nonempty subset of $\mathbb{R}^{p}$, and $Y(x)$ is an infinite index subset of $\mathbb{R}^{q}$ depending on $x$, defined by

$$
Y(x)=\left\{y \in \mathbb{R}^{q} / g(x, y) \leq 0\right\},
$$

where $g: \mathbb{R}^{p} \times \mathbb{R}^{q} \rightarrow \mathbb{R}$ is a function.

Received 18 January 2013; Revised 21 January 2014; Accepted 22 January 2014.

Part of the special issue, "Variational Analysis and Applications."

Address correspondence to Samir Adly, Laboratoire XLIM UMR-CNRS 6172, Département de Mathématiques, Université de Limoges, 123 Avenue Albert Thomas, 87060 Limoges Cedex, France; E-mail: samir.adly@unilim.fr 
Note that semi-infinite programming problems differ from generalized semi-infinite programming problems by the fact that the index set $Y(x)$ does not depend on the decision variable $x$. The class of generalized semi-infinite programming problems has several applications. We cite, for example, approximation theory, economics, optimal control, and engineering.

In this article, we investigate a class of nonsmooth generalized semiinfinite programming problems of the form of $(S)$ whose feasible sets are represented by a reverse convex constraint; that is, a constraint of the form $h(x) \geq 0$, with $h: \mathbb{R}^{p} \rightarrow \mathbb{R}$ is a convex function. So that, in addition to the tools of convex analysis, the use of some results related to reverse convex problems will play an important role in our investigation. Precisely, our goal in this article is to give necessary and sufficient optimality conditions for problem $(S)$. Normal cones, subdifferentials and min-max problems are used to express some of these optimality conditions. In particular, under appropriate assumptions and a new constraint qualification, we provide a necessary and sufficient optimality condition which reduces the problem $(S)$ to a min-max problem constrained with compact convex linked constraints. We note that these optimality conditions are new and do not use differentiability assumptions. For papers dealing with optimality conditions for generalized semi-infinite programming problems, we cite, for example, [1-12].

This article is organized as follows. In section 2, we recall some fundamental results of convex analysis that we need in the sequel. Section 3 is devoted to some preliminaries. In section 4 , we provide necessary and sufficient optimality conditions for the generalized semiinfinite programming problem $(S)$.

\section{BACKGROUND OF CONVEX ANALYSIS}

In this section, we recall some results related to convex analysis that we will use in the sequel.

Definition 2.1. Let $h: \mathbb{R}^{n} \longrightarrow \overline{\mathbb{R}}$ be a function. The set

$$
\operatorname{dom} h=\left\{x \in \mathbb{R}^{n} / h(x)<+\infty\right\}
$$

is called the effective domain of $h$. The function $h$ is said to be proper if $\operatorname{dom} h \neq \emptyset$, and $h(x)>-\infty$, for all $x \in \mathbb{R}^{n}$.

In the sequel, for a subset $A$ of $\mathbb{R}^{n}$,int $A$ and $\operatorname{bd} A$ denote, respectively, the interior and the boundary of $A$.

Proposition 2.1 ([13]). Let $C$ be a nonempty convex subset of $\mathbb{R}^{n}$ with a nonempty interior. Then, $\bar{C}=\overline{\operatorname{int} C}$. 
Proposition 2.2 ([13]). Let $h: \mathbb{R}^{n} \longrightarrow \mathbb{R}$ be a convex function. Assume that there exists $x_{0} \in \mathbb{R}^{n}$ such that $h\left(x_{0}\right)<0$. Then

1) $\operatorname{int}\left\{x \in \mathbb{R}^{n} / h(x) \leq 0\right\}=\left\{x \in \mathbb{R}^{n} / h(x)<0\right\}$,

2) $\operatorname{bd}\left\{x \in \mathbb{R}^{n} / h(x) \leq 0\right\}=\left\{x \in \mathbb{R}^{n} / h(x)=0\right\}$.

Theorem 2.1 ([14]). Let $h: \mathbb{R}^{n} \longrightarrow \mathbb{R} \cup\{+\infty\}$ be a proper convex function. Assume that $\operatorname{int}(\operatorname{dom} h)$ is nonempty. Then, $h$ is continuous on $\operatorname{int}(\operatorname{dom} h)$.

Definition 2.2. Let $h: \mathbb{R}^{n} \longrightarrow \mathbb{R} \cup\{+\infty\}$ be a convex function, $\bar{x} \in$ $\operatorname{dom} h$ and $x^{*} \in \mathbb{R}^{n}$.

1) The vector $x^{*}$ is said to be a subgradient of $h$ at $\bar{x}$ if

$$
h(x) \geq h(\bar{x})+<x^{*}, x-\bar{x}>\quad \forall x \in \mathbb{R}^{n} .
$$

The set of all subgradients of $h$ at $\bar{x}$, which is denoted by $\partial h(\bar{x})$, is called the subdifferential of $h$ at $\bar{x}$, that is, the set defined by

$$
\partial h(\bar{x})=\left\{x^{*} \in \mathbb{R}^{n} / h(x) \geq h(\bar{x})+<x^{*}, x-\bar{x}>, \forall x \in \mathbb{R}^{n}\right\} .
$$

When $\partial h(\bar{x}) \neq \emptyset$, we say that $h$ is subdifferentiable at $\bar{x}$.

2) Let $\epsilon \geq 0$. The vector $x^{*}$ is said to be an $\epsilon$-subgradient of $h$ at $\bar{x}$ if

$$
h(x) \geq h(\bar{x})+<x^{*}, x-\bar{x}>-\epsilon \quad \forall x \in \mathbb{R}^{n} .
$$

The set of all $\epsilon$-subgradients of $h$ at $\bar{x}$, which is denoted by $\partial_{\epsilon} h(\bar{x})$, is called the $\epsilon$-subdifferential of $h$ at $\bar{x}$, that is, the set defined by

$$
\partial_{\epsilon} h(\bar{x})=\left\{x^{*} \in \mathbb{R}^{n} / h(x) \geq h(\bar{x})+<x^{*}, x-\bar{x}>-\epsilon, \forall x \in \mathbb{R}^{n}\right\} .
$$

When $\epsilon=0, \partial_{\epsilon} h(\bar{x})$ reduces to $\partial h(\bar{x})$.

Then, we have the following results concerning the subdifferential.

Theorem 2.2 ([14]). Let $h: \mathbb{R}^{n} \longrightarrow \mathbb{R} \cup\{+\infty\}$ be a proper convex function. Assume that $\operatorname{int}(\operatorname{dom} h)$ is nonempty. Then, for all $x \in \operatorname{int}(\operatorname{dom} h)$, we have $\partial h(x) \neq \emptyset$.

Theorem 2.3 ([13]). Let $f, g: \mathbb{R}^{n} \rightarrow \mathbb{R} \cup\{+\infty\}$ be two convex functions. Assume that there exists $x_{0} \in \operatorname{dom} f \cap \operatorname{dom} g$ such that $g$ is continuous at $x_{0}$. Then, for every $x \in \operatorname{dom} f \cap \operatorname{dom} g$ and $\epsilon \geq 0$, we have

$$
\partial_{\epsilon}(f+g)(x)=\bigcup_{\substack{\epsilon_{1}, \epsilon_{2} \geq 0 \\ \epsilon_{1}+\epsilon_{2}=\epsilon}}\left\{\partial_{\epsilon_{1}} f(x)+\partial_{\epsilon_{2}} g(x)\right\}
$$


Definition 2.3. Let $C$ be a nonempty convex subset of $\mathbb{R}^{n}$ and $\bar{x} \in C$. The normal cone of $C$ at $\bar{x}$, denoted by $\mathcal{N}(C, \bar{x})$ is defined by

$$
\mathcal{N}(C, \bar{x})=\left\{x^{*} \in \mathbb{R}^{n} /<x^{*}, x-\bar{x}>\leq 0, \forall x \in C\right\} .
$$

Definition 2.4. Let $C$ be a nonempty convex subset of $\mathbb{R}^{n}$ and $\bar{x} \in C$. Let $\epsilon \geq 0$. The set of $\epsilon$-normal directions to $C$ at $\bar{x}$, denoted by $\mathcal{N}_{\epsilon}(C, \bar{x})$ is defined by

$$
\mathcal{N}_{\epsilon}(C, \bar{x})=\left\{x^{*} \in \mathbb{R}^{n} /<x^{*}, x-\bar{x}>\leq \epsilon, \forall x \in C\right\} .
$$

When $\epsilon=0$, then $\mathcal{N}_{\epsilon}(C, \bar{x})$ reduces to $\mathcal{N}(C, \bar{x})$.

Definition 2.5. Let $A$ be a subset of $\mathbb{R}^{n}$. The indicator function of $A$ denoted by $\psi_{A}$ is the function defined on $\mathbb{R}^{n}$ by

$$
\psi_{A}(x)= \begin{cases}0 & \text { if } x \in A \\ +\infty & \text { if } x \notin A .\end{cases}
$$

Proposition 2.3 ([13]). Let $C$ be a closed convex subset of $\mathbb{R}^{n}$. Let $x \in$ bd $C$. Then, there exists $x^{*} \in \mathbb{R}^{n} \backslash\{0\}$, such that $x^{*} \in \mathcal{N}(C, x)$.

Theorem 2.4 ([13]). Let $C$ and $D$ be two nonempty convex subsets of $\mathbb{R}^{n}$. Assume that $C \cap$ int $D \neq \emptyset$. Let $x \in C \cap D$. Then

$$
\mathcal{N}_{C \cap D}(x)=\mathcal{N}_{C}(x)+\mathcal{N}_{D}(x) .
$$

In the sequel, for a function $h: \mathbb{R}^{n} \longrightarrow \mathbb{R}$ and $\alpha \in \mathbb{R}, \mathscr{L}_{\alpha}(h)$ will denote the lower level set of $h$ at $\alpha$, that is,

$$
\mathscr{L}_{\alpha}(h)=\left\{y \in \mathbb{R}^{n} / h(y) \leq \alpha\right\} .
$$

Then, we have the following results.

Theorem 2.5 ([13]). Let $h: \mathbb{R}^{n} \longrightarrow \mathbb{R}$ be a convex function. Let $x \in \mathbb{R}^{n}$ such that $0 \notin \partial h(x)$. Then

$$
\mathcal{N}\left(\mathscr{L}_{h(x)}(h), x\right)=\mathbb{R}^{+} \partial h(x)
$$

where $\mathbb{R}^{+} \partial h(x)=\left\{\lambda x^{*} / \lambda \in \mathbb{R}^{+}, x^{*} \in \partial h(x)\right\}$. 
Theorem $2.6([13])$. Let $h: \mathbb{R}^{n} \longrightarrow \mathbb{R}$ be a convex function and $\alpha \in \mathbb{R}$. Assume that there exists $\bar{x} \in \mathbb{R}^{n}$ such that $h(\bar{x})<\alpha$. Then, for any $x \in$ $\operatorname{bd}\left(\mathscr{L}_{\alpha}(h)\right)$, we have

$$
\mathcal{N}_{\epsilon}\left(\mathscr{L}_{\alpha}(h), x\right)=\bigcup_{\beta \geq 0} \partial_{\epsilon}(\beta h)(x) .
$$

Theorem 2.7 ([15]). Consider the following convex maximization problem

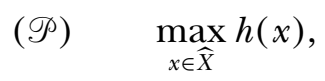

where $h: \mathbb{R}^{n} \rightarrow \mathbb{R}$ is a convex function and $\widehat{X}$ is a closed convex subset of $\mathbb{R}^{n}$. Let $\hat{x} \in \widehat{X}$ be a local maximum of $(\mathscr{P})$. Then

$$
\partial h(\hat{x}) \subset \mathcal{N}(\widehat{X}, \hat{x}) .
$$

\section{PRELIMINARIES}

In this section, we give some preliminary results that will be useful for our study. For the convenience of the reader, let us first recall the following definition.

Definition 3.1. Consider the following minimization problem

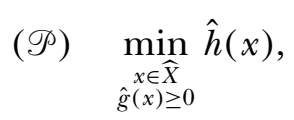

where $\hat{h}: \mathbb{R}^{p} \rightarrow \mathbb{R}$ is a function, $\hat{g}: \mathbb{R}^{p} \rightarrow \mathbb{R}$ is a convex function and $\widehat{X}$ is a nonempty subset of $\mathbb{R}^{p}$. The constraint $g(x) \geq 0$ is called a reverse convex constraint and the problem $(\mathscr{P})$ is called a problem with a reverse convex constraint. It follows that the complement of the set $\left\{x \in \mathbb{R}^{p} / g(x) \geq 0\right\}$ is open and convex. If, moreover, $\hat{h}$ and $\widehat{X}$ are convex, $(\mathscr{P})$ is called a reverse convex problem. The constraint $g(x) \geq 0$ is called essential if

$$
\inf _{x \in \widehat{X}} \hat{h}(x)<\inf _{\substack{x \in \widehat{X} \\ \hat{g}(x) \geq 0}} \hat{h}(x) .
$$

Note that when this property is not satisfied, the problem $(\mathscr{P})$ is reduced to a convex programming problem.

In the sequel, and under appropriate assumptions, the generalized semi-infinite programming problem $(S)$ will be viewed as a reverse convex problem. Then, we will make the following assumptions. 
(3.1) The set $X$ is convex and compact,

(3.2) The functions $F$ is convex on $\mathbb{R}^{p}$,

(3.3) The functions $G$ is concave on $\mathbb{R}^{p} \times \mathbb{R}^{q}$,

(3.4) The function $g$ is convex on $\mathbb{R}^{p} \times \mathbb{R}^{q}$,

(3.5) $\inf _{x \in X} F(x)<\inf (S)$, where $\inf (S)$ denotes the infimal value of problem $(S)$,

(3.6) There exists a convex compact subset $Z$ of $\mathbb{R}^{q}$, such that $Y(x) \subset Z$, for any $x \in X$.

For $(x, y) \in \mathbb{R}^{p} \times \mathbb{R}^{q}$, set

$$
f(x, y)=-G(x, y) \quad \text { and } \quad v(x)=\inf _{y \in Y(x)} f(x, y) .
$$

Then, the problem $(S)$ is written as

$$
\text { (S) } \min _{\substack{x \in X \\ v(x) \geq 0}} F(x, y) .
$$

Remark 3.1. Let Assumptions (3.1)-(3.5) hold. Assumptions (3.3) and (3.4) imply that the marginal function $v($.$) is convex, and Assumption$ (3.5) implies that there exists $\bar{x} \in X$ verifying

$$
F(\bar{x})<\inf (S) \text { and } \quad v(\bar{x})<0 .
$$

So that, in this case the constraint $v(x) \geq 0$ is essential. Furthermore, $(S)$ is a reverse convex problem. This leads to the following definition.

Definition 3.2. Assume that $X$ is convex, $F$ and $g$ are convex functions on $\mathbb{R}^{p}$ and $\mathbb{R}^{p} \times \mathbb{R}^{q}$, respectively, and $G$ is concave on $\mathbb{R}^{p} \times \mathbb{R}^{q}$. We call the problem $(S)$ a reverse convex generalized semi-infinite programming problem.

Remark 3.2. Let Assumptions (3.3), (3.4), and (3.6) hold. Then, the marginal function $v($.$) is convex and finite valued. Hence, it is a$ continuous function on $\mathbb{R}^{p}$ (Theorem 2.1). If, moreover, Assumptions (3.1) and (3.2) are satisfied, then, the problem $(S)$ admits at least one solution.

Consider the following minimization problem

$$
\text { (Q) } \min _{\substack{x \in X \\ v(x)=0}} F(x) .
$$

Then, we have the following result. 
Proposition 3.1. Let Assumptions (3.1)-(3.6) hold. Then, the problems (S) and (Q) are equivalent in the following sense

i) (S) and (Q) have the same set of solutions,

ii) $\inf (S)=\inf (\mathscr{Q})$.

Proof. Let $\bar{x} \in X$ be the point given in Remark 3.1 which satisfies

$$
F(\bar{x})<\inf (S) \text { and } \quad v(\bar{x})<0 .
$$

i) Let $\hat{x}$ be a solution of problem $(S)$. Note that such a point exists according to Remark 3.2. Let us show that $v(\hat{x})=0$. Assume that $v(\hat{x})>0$. Then, $\hat{x} \neq \bar{x}$, and from the continuity of the function $v($.$) , there exists \tilde{x} \in$ ]$\hat{x}, \bar{x}[, \tilde{x}=t \bar{x}+(1-t) \hat{x}, t \in] 0,1[$, verifying $v(\tilde{x})=0$. Hence, $\tilde{x}$ is a feasible point of $(S)$. Then, from the convexity of $F$, we have

$$
F(\tilde{x}) \leq t F(\bar{x})+(1-t) F(\hat{x})<F(\hat{x})
$$

which contradicts the optimality of $\hat{x}$ to the problem $(S)$. To show that $\hat{x}$ solves (Q), let $x$ be a feasible point of problem (Q). Then, $x$ is a feasible point of $(S)$. Hence,

$$
F(\hat{x}) \leq F(x)
$$

So that $\hat{x}$ solves (Q).

Conversely, let $\hat{x}$ be a solution of (Q). Then $v(\hat{x})=0$. Hence,

$$
\inf (S) \leq F(\hat{x})
$$

Let $x$ be a feasible point of $(S)$. We distinguish the following cases.

1) Assume that $v(x)=0$. Hence, $x$ is a feasible point of problem (Q). So that $F(\hat{x}) \leq F(x)$.

2) Assume that $v(x)>0$. Using (1) and that the marginal function $v($.$) is continuous on \mathbb{R}^{p}$, it follows that there exists $\left.x^{*} \in\right] x, \bar{x}\left[, x^{*}=t \bar{x}+\right.$ $(1-t) x, t \in] 0,1\left[\right.$ verifying $v\left(x^{*}\right)=0$. Since $X$ is convex, then $x^{*} \in X$. So that $x^{*}$ is a feasible point of (Q). It follows that

$$
F(\hat{x}) \leq F\left(x^{*}\right)
$$

Assume that $F(\hat{x})>F(x)$. From the convexity of $F$, we have

$$
F\left(x^{*}\right) \leq t F(\bar{x})+(1-t) F(x)<t \inf (S)+(1-t) F(\hat{x}) .
$$


Using (2), we obtain

$$
F\left(x^{*}\right)<F(\hat{x})
$$

which contradicts (3). It follows that

$$
F(\hat{x}) \leq F(x) .
$$

Therefore, from the two cases, we deduce that $\hat{x}$ is a solution of $(S)$.

ii) We have

$$
\{x \in X / v(x)=0\} \subset\{x \in X / v(x) \geq 0\} .
$$

So that

$$
\inf (S) \leq \inf (\mathscr{Q})
$$

Let $x_{1}$ and $x_{2}$ be solutions to problems $(S)$ and (Q), respectively. From the above case i), we have $v\left(x_{1}\right)=0$. So that, $x_{1}$ is a feasible point of (Q) $)$. It follows that

$$
F\left(x_{2}\right)=\inf (\mathscr{Q}) \leq F\left(x_{1}\right)=\inf (S),
$$

where the inequality follows from the feasibility of $x_{1}$ to problem (Q). We deduce that $\inf (S)=\inf (\mathscr{Q})$.

Remark 3.3. Note that a necessary condition for a feasible point $x$ of $(S)$ to be optimal is that $v(x)=0$ [see the proof of i) of Proposition 3.1].

\section{OPTIMALITY CONDITIONS}

In this section, we provide necessary and sufficient optimality conditions for the generalized semi-infinite programming problem $(S)$. Some of these optimality conditions are established via stability results.

Theorem 4.1. (Necessary and Sufficient Optimality Conditions) Let Assumptions (3.1)-(3.6) hold. Let $\hat{x}$ be a feasible point of problem $(S)$. Then, $\hat{x}$ is a solution of $(S)$ if and only if

i) $v(\hat{x})=0$,

ii) $X \cap \operatorname{int}\left(\mathscr{L}_{F(\hat{x})}(F)\right) \subset X \cap \operatorname{int}\left(\mathscr{L}_{v(\hat{x})}(v)\right)$.

Proof. Let $\bar{x}$ be the point given in Remark 3.1 that satisfies

$$
F(\bar{x})<\inf (S) \text { and } \quad v(\bar{x})<0 .
$$


$(\Longrightarrow)$ Assume that $\hat{x}$ is a solution of $(S)$. Then, $v(\hat{x})=0$ (see Remark 3.3). We have

$$
F(\bar{x})<\inf (S)=F(\hat{x}) \text { and } v(\bar{x})<0=v(\hat{x}) .
$$

With the above two strict inequalities and the convexity of $F$ and $v$, we deduce that

$$
\operatorname{int}\left(\mathscr{L}_{F(\hat{x})}(F)\right)=\left\{x \in \mathbb{R}^{p} / F(x)<F(\hat{x})\right\}
$$

and

$$
\begin{aligned}
\operatorname{int}\left(\mathscr{L}_{v(\hat{x})}(v)\right) & =\left\{x \in \mathbb{R}^{p} / v(x)<v(\hat{x})\right\} \\
& =\left\{x \in \mathbb{R}^{p} / v(x)<0\right\} .
\end{aligned}
$$

Assume that $X \cap \operatorname{int}\left(\mathscr{L}_{F(\hat{x})}(F)\right)$ is not a subset of $X \cap \operatorname{int}\left(\mathscr{L}_{v(\hat{x})}(v)\right)$. Then, there exists $\tilde{x} \in X$ such that $F(\tilde{x})<F(\hat{x})$, but

$$
v(\tilde{x}) \geq v(\hat{x})=0
$$

that is, $\tilde{x} \notin \operatorname{int}\left(\mathscr{L}_{v(\hat{x})}(v)\right)$. Hence, $\tilde{x}$ is a feasible point of $(S)$. This contradicts the optimality of $\hat{x}$.

$(\Longleftarrow)$ Let the conditions i) and ii) be satisfied. Assume that there exists $x \in X$ such that

$$
v(x) \geq 0 \quad \text { and } \quad F(x)<F(\hat{x})
$$

that is, $\hat{x}$ is not a solution of $(S)$. Then we have $x \in X \cap \operatorname{int}\left(\mathscr{L}_{F(\hat{x})}(F)\right)$. Property ii) implies that

$$
v(x)<v(\hat{x})
$$

Since from i) we have $v(\hat{x})=0$, it follows that $v(x)<0$, which contradicts the first inequality in (4). So that $\hat{x}$ is a solution of $(S)$.

From Theorem 4.1 we deduce the following necessary optimality condition expressed in terms of normal cones and subdifferentials.

Corollary 4.1. Let Assumptions (3.1)-(3.6) hold. Let $\hat{x}$ be a feasible point of problem $(S)$. Assume that $\hat{x}$ is a solution of $(S)$. Then, $v(\hat{x})=0$ and for any $\epsilon \geq 0$, we have

$$
\bigcup_{\substack{\alpha_{1}, \alpha_{2} \geq 0 \\ \alpha_{1}+\alpha_{2}=\epsilon}}\left\{\mathcal{N}_{\alpha_{1}}(X, \hat{x})+\bigcup_{\alpha \geq 0} \partial_{\alpha_{2}}(\alpha v)(\hat{x})\right\} \subset \bigcup_{\substack{\beta_{1}, \beta_{2} \geq 0 \\ \beta_{1}+\beta_{2}=\epsilon}}\left\{\mathcal{N}_{\beta_{1}}(X, \hat{x})+\bigcup_{\beta \geq 0} \partial_{\beta_{2}}(\alpha v)(\hat{x})\right\} .
$$


Proof. Since $\hat{x}$ solves $(S)$, it follows from Theorem 4.1 that $v(\hat{x})=0$ and

$$
X \cap \operatorname{int}\left(\mathscr{L}_{F(\hat{x})}(F)\right) \subset X \cap \operatorname{int}\left(\mathscr{L}_{v(\hat{x})}(v)\right) .
$$

Then

$$
\begin{aligned}
\overline{\operatorname{int} X \cap \operatorname{int}\left(\mathscr{L}_{F(\hat{x})}(F)\right)} \subset \overline{X \cap \operatorname{int}\left(\mathscr{L}_{F(\hat{x})}(F)\right)} & \subset \overline{X \cap \operatorname{int}\left(\mathscr{L}_{v(\hat{x})}(v)\right)} \\
& \subset \overline{X \cap \mathscr{L}_{v(\hat{x})}(v)} \\
& =X \cap \mathscr{L}_{v(\hat{x})}(v)
\end{aligned}
$$

where the above equality follows from the closedness of the sets $X$ and $\mathscr{L}_{v(\hat{x})}(v)$. Moreover,

$$
\begin{aligned}
\overline{\operatorname{int} X \cap \operatorname{int}\left(\mathscr{L}_{F(\hat{x})}(F)\right)} & =\overline{\operatorname{int}\left(X \cap \mathscr{L}_{F(\hat{x})}(F)\right)} \\
& =\overline{X \cap \mathscr{L}_{F(\hat{x})}(F)} \\
& =X \cap \mathscr{L}_{F(\hat{x})}(F)
\end{aligned}
$$

where the above second equality follows from the convexity of the set $X \cap$ $\mathscr{L}_{F(\hat{x})}(F)$ (see Proposition 2.1). Therefore, from (5) we obtain

$$
X \cap \mathscr{L}_{F(\hat{x})}(F) \subset X \cap \mathscr{L}_{v(\hat{x})}(v) .
$$

So that, for any $\epsilon \geq 0$, we have

$$
\mathcal{N}_{\epsilon}\left(X \cap \mathscr{L}_{v(\hat{x})}(v)\right) \subset \mathcal{N}_{\epsilon}\left(X \cap \mathscr{L}_{F(\hat{x})}(F)\right) .
$$

Let $\bar{x} \in X$ be the point given in Remark 3.1. Then, $\bar{x}$ satisfies

$$
F(\bar{x})<F(\hat{x}) \quad \text { and } \quad v(\bar{x})<v(\hat{x}) .
$$

So that

$$
X \cap \operatorname{int}\left(\mathscr{L}_{F(\hat{x})}(F)\right) \neq \emptyset \quad \text { and } \quad X \cap \operatorname{int}\left(\mathscr{L}_{v(\hat{x})}(v)\right) \neq \emptyset .
$$

Then, we have

$$
\operatorname{dom} \psi_{X} \cap \operatorname{int}\left(\operatorname{dom} \psi_{\mathscr{I}_{F(\hat{x})}(F)}\right) \neq \emptyset
$$

and

$$
\operatorname{dom} \psi_{X} \cap \operatorname{int}\left(\operatorname{dom} \psi_{\Phi_{v(\hat{x})}(v)}\right) \neq \emptyset .
$$


On the other hand, we have

$$
\begin{aligned}
\mathcal{N}_{\epsilon}\left(X \cap \mathscr{L}_{F(\hat{x})}(F), \hat{x}\right) & =\partial_{\epsilon} \psi_{X \cap \mathscr{L}_{F(\hat{x})}(F)}(\hat{x}) \\
& =\partial_{\epsilon}\left(\psi_{X}+\psi_{\mathscr{L}_{F(\hat{x})}(F)}\right)(\hat{x}) .
\end{aligned}
$$

Using Theorems 2.1 and 2.3, we obtain

$$
\partial_{\epsilon}\left(\psi_{X}+\psi_{\mathscr{L}_{F(\hat{x})}(F)}\right)(\hat{x})=\bigcup_{\substack{\beta_{1}, \beta_{2} \geq 0 \\ \beta_{1}+\beta_{2}=\epsilon}}\left\{\partial_{\beta_{1}} \psi_{X}(\hat{x})+\partial_{\beta_{2}} \psi_{\mathscr{L}_{F(\hat{x})}(F)}(\hat{x})\right\}
$$

On the other hand, since $\operatorname{int}\left(\mathscr{L}_{F(\hat{x})}(F)\right)$ is nonempty and $\hat{x} \in \operatorname{bd}\left(\mathscr{L}_{F(\hat{x})}(F)\right)$, it follows that (see Theorem 2.6)

$$
\begin{aligned}
\partial_{\beta_{2}} \psi_{\mathscr{L}_{F(\hat{x})}(F)}(\hat{x}) & =\mathcal{N}_{\beta_{2}}\left(\mathscr{L}_{F(\hat{x})}(F), \hat{x}\right) \\
& =\bigcup_{\beta \geq 0} \partial_{\beta_{2}}(\beta F)(\hat{x}) .
\end{aligned}
$$

Hence,

$$
\partial_{\epsilon}\left(\psi_{X}+\psi_{\mathscr{L}_{F(\hat{x})}(F)}\right)(\hat{x})=\bigcup_{\substack{\beta_{1}, \beta_{2} \geq 0 \\ \beta_{1}+\beta_{2}=\epsilon}}\left\{\mathcal{N}_{\beta_{1}}(X, \hat{x})+\bigcup_{\beta \geq 0} \partial_{\beta_{2}}(\beta F)(\hat{x})\right\} .
$$

By similar calculation and arguments, we also have

$$
\mathcal{N}_{\epsilon}\left(X \cap \mathscr{L}_{v(\hat{x})}(v), \hat{x}\right)=\bigcup_{\substack{\alpha_{1}, \alpha_{2} \geq 0 \\ \alpha_{1}+\alpha_{2}=\epsilon}}\left\{\mathcal{N}_{\alpha_{1}}(X, \hat{x})+\bigcup_{\alpha \geq 0} \partial_{\alpha_{2}}(\alpha v)(\hat{x})\right\} .
$$

Then, the result follows from the inclusion in (6).

In the remainder of this section, we will give optimality conditions involving stability results. For $\alpha \in \mathbb{R}$, we consider the following parameterized maximization problem

$$
\left(\mathscr{R}_{\alpha}\right) \quad \max _{\substack{x \in X \\ F(x) \leq \alpha}} v(x)
$$

and for $\beta \in \mathbb{R}$, we associate to the generalized semi-infinite programming problem $(S)$ the following parameterized minimization problem

$$
\left(S_{\beta}\right) \quad \min _{\substack{x \in X \\ v(x) \geq \beta}} F(x) .
$$


Let

$$
\mathscr{F}\left(\mathscr{R}_{\alpha}\right)=\{x \in X / F(x) \leq \alpha\} \quad \text { and } \quad \mathscr{F}\left(S_{\beta}\right)=\{x \in X / v(x) \geq \beta\}
$$

be, respectively, the feasible sets of problems $\left(\mathscr{R}_{\alpha}\right)$ and $\left(S_{\beta}\right)$ respectively. Note that for $\beta=0$, we obtain the problem $(S)$.

The following Propositions 4.1 and 4.2 give stability results for problems $\left(\mathscr{R}_{\alpha}\right)$ and $\left(S_{\beta}\right)$, respectively. Their proofs are an adaptation of those given in [16]. These stability results allow us to establish a necessary and sufficient optimality condition that reduces the problem $(S)$ to a minmax problem. Then, in order to provide the main result of this section, we need first to establish some preliminary results.

Proposition 4.1. Let $\alpha \in \mathbb{R}$. Assume that Assumptions (3.1)-(3.4) and (3.6) hold, and that $\alpha$ is not a local minimum of $F$ over $X$. Then, for any sequence $\alpha_{m} \rightarrow \alpha$, as $m \rightarrow+\infty$, with $\alpha_{m}<\alpha, \forall m \in \mathbb{N}$, we have

$$
\lim _{m \rightarrow+\infty} \sup \left(\mathscr{R}_{\alpha_{m}}\right)=\sup \left(\mathscr{R}_{\alpha}\right)
$$

Proof. Let $\epsilon_{k} \rightarrow 0^{+}, k \in \mathbb{N}$. For $\epsilon_{k}$, there exists $x_{k} \in X$, such that

$$
F\left(x_{k}\right) \leq \alpha \quad \text { and } \quad v\left(x_{k}\right)>\sup \left(\mathscr{R}_{\alpha}\right)-\epsilon_{k}
$$

We distinguish the following cases.

Case 1. Assume that there exists $k_{0} \in \mathbb{N}$, such that

$$
F\left(x_{k}\right)=\alpha \quad \forall k \in \mathbb{N}, k \geq k_{0} .
$$

Then, since $\alpha$ is not a local minimum of $F$ over $X$, for $\frac{1}{p}$, there exists $x_{k, p} \in$ $B\left(x_{k}, \frac{1}{p}\right) \cap X$, such that

$$
F\left(x_{k, p}\right)<F\left(x_{k}\right)=\alpha \quad \forall k \geq k_{0}
$$

We have $x_{k, p} \rightarrow x_{k}$, as $p \rightarrow+\infty$. Then, from the continuity of $F$ as a finite convex function, we have $F\left(x_{k, p}\right) \rightarrow F\left(x_{k}\right)$, as $p \rightarrow+\infty$. Moreover, $\alpha_{m} \rightarrow \alpha$, as $m \rightarrow+\infty$, and $\alpha_{m}<\alpha, \forall m \in \mathbb{N}$. Then, there exists $m_{0} \in \mathbb{N}$, such that

$$
F\left(x_{k, p}\right)<\alpha_{m}, \quad \forall m \geq m_{0}
$$

Since $x_{k, p}$ is a feasible point of problem $\left(\mathscr{R}_{\alpha_{m}}\right), m \geq m_{0}$, we have

$$
\sup \left(\mathscr{R}_{\alpha_{m}}\right) \geq v\left(x_{k, p}\right) \quad m \geq m_{0} .
$$


Then

$$
\liminf _{m \rightarrow+\infty} \sup \left(\mathscr{R}_{\alpha_{m}}\right) \geq v\left(x_{k, p}\right) .
$$

Since $v($.$) is lower semi-continuous on \mathbb{R}^{p}$, then

$$
\liminf _{m \rightarrow+\infty} \sup \left(\mathscr{R}_{\alpha_{m}}\right) \geq \liminf _{p \rightarrow+\infty} v\left(x_{k, p}\right) \geq v\left(x_{k}\right) \geq \sup \left(\mathscr{R}_{\alpha}\right)-\epsilon_{k}
$$

Letting $k \rightarrow+\infty$, we obtain

$$
\liminf _{m \rightarrow+\infty} \sup \left(\mathscr{R}_{\alpha_{m}}\right) \geq \sup \left(\mathscr{R}_{\alpha}\right) .
$$

On the other hand, for every $m \in \mathbb{N}$, we have $\mathscr{F}\left(\mathscr{R}_{\alpha_{m}}\right) \subset \mathscr{F}\left(\mathscr{R}_{\alpha}\right)$, and, hence,

$$
\sup \left(\mathscr{R}_{\alpha_{m}}\right) \leq \sup \left(\mathscr{R}_{\alpha}\right)
$$

So that

$$
\limsup _{m \rightarrow+\infty} \sup \left(\mathscr{R}_{\alpha_{m}}\right) \leq \sup \left(\mathscr{R}_{\alpha}\right)
$$

From (7) and (8), we deduce that

$$
\lim _{m \rightarrow+\infty} \sup \left(\mathscr{R}_{\alpha_{m}}\right)=\sup \left(\mathscr{R}_{\alpha}\right)
$$

Case 2. Assume that there exists an infinite subset $\mathcal{N}$ of $\mathbb{N}$ such that

$$
F\left(x_{k}\right) \neq \alpha \quad \forall k \in \mathcal{N}
$$

So that

$$
F\left(x_{k}\right)<\alpha \quad \forall k \in \mathcal{N} .
$$

Then, for all $k \in \mathcal{N}$, we have

$$
F\left(x_{k}\right)<\alpha_{m} \quad \text { for large } m \text {. }
$$

It follows from the feasibility of $x_{k}$ to problem $\left(\mathscr{R}_{\alpha_{m}}\right)$ that

$$
\sup \left(\mathscr{R}_{\alpha_{m}}\right) \geq v\left(x_{k}\right)>\sup \left(\mathscr{R}_{\alpha}\right)-\epsilon_{k}
$$

Then

$$
\liminf _{m \rightarrow+\infty} \sup \left(\mathscr{R}_{\alpha_{m}}\right) \geq \sup \left(\mathscr{R}_{\alpha}\right)-\epsilon_{k} .
$$


Letting $k \rightarrow+\infty, k \in \mathcal{N}$, we obtain

$$
\liminf _{m \rightarrow+\infty} \sup \left(\mathscr{R}_{\alpha_{m}}\right) \geq \lim _{\substack{k \rightarrow+\infty \\ k \in \mathcal{N}}}\left(\sup \left(\mathscr{R}_{\alpha}\right)-\epsilon_{k}\right)=\sup \left(\mathscr{R}_{\alpha}\right) .
$$

On the other hand, we have

$$
\sup \left(\mathscr{R}_{\alpha}\right) \geq \sup \left(\mathscr{R}_{\alpha_{m}}\right)
$$

Then

$$
\sup \left(\mathscr{R}_{\alpha}\right) \geq \limsup _{m \rightarrow+\infty} \sup \left(\mathscr{R}_{\alpha_{m}}\right)
$$

From (9) and (10), we obtain

$$
\lim _{m \rightarrow+\infty} \sup \left(\mathscr{R}_{\alpha_{m}}\right)=\sup \left(\mathscr{R}_{\alpha}\right)
$$

The following theorem provides a necessary optimality condition for $(S)$.

Theorem 4.2. Assume that Assumptions (3.1)-(3.6) are satisfied. Assume that $\hat{x}$ is a solution of $(S)$. Then, $v(\hat{x})=0$ and

$$
\inf _{\substack{x \in X \\ F(x) \leq F(\hat{x})}} \sup _{\substack{y \in \mathbb{R}^{q} \\ g(x, y) \leq 0}} G(x, y)=0 .
$$

Proof. The condition $v(\hat{x})=0$ follows from Theorem 4.1. Let $\alpha=F(\hat{x})$, and $\alpha_{m} \rightarrow \alpha$, as $m \rightarrow+\infty$, with $\alpha_{m}<\alpha, \forall m \in \mathbb{N}$. Let $x \in X$ such that $F(x) \leq \alpha_{m}$. Then

$$
v(x)<0
$$

Otherwise $v(x) \geq 0$. So that

$$
\alpha_{m}<\alpha=F(\hat{x})=\inf (S) \leq F(x) \leq \alpha_{m}
$$

which gives a contradiction. Then, from (11), we have

$$
\sup _{\substack{x \in X \\ F(x) \leq \alpha_{m}}} v(x)=\sup \left(\mathscr{R}_{\alpha_{m}}\right) \leq 0 .
$$

Proposition 4.1 implies that

$$
\lim _{m \rightarrow+\infty} \sup \left(\mathscr{R}_{\alpha_{m}}\right)=\sup \left(\mathscr{R}_{\alpha}\right) \leq 0
$$


Since $v(\hat{x})=0$ and $\hat{x}$ is a feasible point of problem $\left(\mathscr{R}_{\alpha}\right)$, it follows that

$$
\begin{aligned}
\sup \left(\mathscr{R}_{\alpha}\right)= & \sup _{\substack{x \in X \\
F(x) \leq F(\hat{x})}} v(x)=0 \\
= & \sup _{\substack{x \in X \\
F(x) \leq F(\hat{x})}} \inf _{y \in Y(x)} f(x, y)
\end{aligned}
$$

with $f(x, y)=-G(x, y)$. So that

$$
\inf _{\substack{x \in X \\ F(x) \leq F(\hat{x})}} \sup _{\substack{y \in \mathbb{R} q \\ g(x, y) \leq 0}} G(x, y)=0 .
$$

We consider the following constraint qualification:

(4.1) For every $x \in X$ verifying $v(x)=0$, there exist $x^{*} \in \partial v(x)$ and $\hat{x} \in X$, such that $\left\langle x^{*}, \hat{x}-x\right\rangle>0$.

Remark 4.1. Assumption (4.1) is equivalent to say that for every $x \in X$ verifying $v(x)=0$, we have $\partial v(x) \not \subset \mathcal{N}(X, x)$. In other terms, if $M$ denotes the set of solutions to the problem

$$
\max _{x \in X} v(x)
$$

we have

$$
\{x \in X / v(x)=0\} \cap M=\emptyset .
$$

Proposition 4.2. Let Assumption (3.1)-(3.6) and (4.1) hold. Let $\beta_{m} \rightarrow 0^{+}$, as $m \rightarrow+\infty$, with $\beta_{m}>0, \forall m \in \mathbb{N}$. Then

$$
\lim _{m \rightarrow+\infty} \inf \left(S_{\beta_{m}}\right)=\inf (S)
$$

Proof. Let $\epsilon_{k} \rightarrow 0^{+}, k \in \mathbb{N}$. For $\epsilon_{k}$, there exists $x_{k} \in X$ such that

$$
v\left(x_{k}\right) \geq 0 \text { and } F\left(x_{k}\right)<\inf (S)+\epsilon_{k} .
$$

We distinguish the following cases.

Case 1. Assume that there exists $k_{0} \in \mathbb{N}$, such that

$$
v\left(x_{k}\right)=0 \quad \forall k \geq k_{0} .
$$

Assumption (4.1) means that for every $x \in X$ such that $v(x)=0, \partial v(x)$ is not a subset of $\mathcal{N}(X, x)$. So that 0 is not a local maximum of $v($.$) over X$ 
(Theorem 2.7). Then, for $\frac{1}{p}, p \in \mathbb{N}^{*}$, there exists $x_{k, p} \in B\left(x_{k}, \frac{1}{p}\right) \cap X$, such that

$$
v\left(x_{k, p}\right)>v\left(x_{k}\right)=0 .
$$

Then, there exists $m_{0} \in \mathbb{N}$, such that

$$
v\left(x_{k, p}\right)>\beta_{m} \quad \forall m \geq m_{0} .
$$

Since $x_{k, p}$ is feasible for $\left(S_{\beta_{m}}\right), m \geq m_{0}$, then

$$
\inf \left(S_{\beta_{m}}\right) \leq F\left(x_{k, p}\right) .
$$

So that

$$
\limsup _{m \rightarrow+\infty} \inf \left(S_{\beta_{m}}\right) \leq F\left(x_{k, p}\right) .
$$

Since $F$ is upper semi-continuous on $\mathbb{R}^{p}$ and $x_{k, p} \rightarrow x_{k}$, as $p \rightarrow+\infty$, then

$$
\limsup _{m \rightarrow+\infty} \inf \left(S_{\beta_{m}}\right) \leq \limsup _{p \rightarrow+\infty} F\left(x_{k, p}\right) \leq F\left(x_{k}\right)<\inf (S)+\epsilon_{k} .
$$

Letting $k \rightarrow+\infty$, we obtain

$$
\limsup _{m \rightarrow+\infty} \inf \left(S_{\beta_{m}}\right) \leq \inf (S) .
$$

On the other hand, we have

$$
\mathscr{F}\left(S_{\beta_{m}}\right) \subset\left\{x \in \mathbb{R}^{p} / x \in X, v(x) \geq 0\right\} .
$$

Then

$$
\inf (S) \leq \inf \left(S_{\beta_{m}}\right)
$$

and

$$
\inf (S) \leq \liminf _{m \rightarrow+\infty} \inf \left(S_{\beta_{m}}\right) .
$$

From (12) and (13), we deduce that

$$
\lim _{m \rightarrow+\infty} \inf \left(\mathscr{S}_{\beta_{m}}\right)=\inf (S) .
$$

Case 2. Assume that there exists an infinite subset $\mathcal{N}$ of $\mathbb{N}$ such that

$$
v\left(x_{k}\right) \neq 0 \quad \forall k \in \mathcal{N} .
$$


So that

$$
v\left(x_{k}\right)>0 \quad \forall k \in \mathcal{N} .
$$

Since $\beta_{m} \rightarrow 0^{+}$, there exists $m_{0} \in \mathbb{N}$ such that for all $k \in \mathcal{N}$, we have

$$
v\left(x_{k}\right)>\beta_{m} \quad \forall m \geq m_{0} .
$$

Hence, $x_{k}$ is a feasible point for $\left(S_{\beta_{m}}\right), m \geq m_{0}$. Then

$$
\inf \left(S_{\beta_{m}}\right) \leq F\left(x_{k}\right)<\inf (S)+\epsilon_{k} .
$$

So that

$$
\limsup _{m \rightarrow+\infty} \inf \left(S_{\beta_{m}}\right) \leq F\left(x_{k}\right)<\inf (S)+\epsilon_{k}
$$

Letting $k$ to $+\infty, k \in \mathcal{N}$, we obtain

$$
\limsup _{m \rightarrow+\infty} \inf \left(S_{\beta_{m}}\right) \leq \lim _{\substack{k \rightarrow+\infty \\ k \in \mathcal{N}}}\left(\inf (S)+\epsilon_{k}\right)=\inf (S)
$$

On the other hand, we have

$$
\inf (S) \leq \inf \left(S_{\beta_{m}}\right)
$$

So that

$$
\inf (S) \leq \liminf _{m \rightarrow+\infty} \inf \left(S_{\beta_{m}}\right) .
$$

Hence, from (14) and (15), we obtain

$$
\lim _{m \rightarrow+\infty} \inf \left(S_{\beta_{m}}\right)=\inf (S)
$$

The following theorem gives a sufficient optimality condition. The proof is based on the use of the stability of problem $(S)$ under perturbation (Proposition 4.2).

Theorem 4.3. Let Assumptions (3.1)-(3.6) and (4.1) hold. Let $\bar{x}$ be a feasible point of $(S)$. Assume that $v(\hat{x})=0$ and $\hat{x}$ satisfies the condition $\mathscr{C}(\hat{x})$ given in Theorem 4.2. Then, $\hat{x}$ solves $(S)$.

Proof. Let $\beta_{m} \rightarrow 0$, with $\beta_{m}>0, \forall m \in \mathbb{N}$. Let $x \in X$ such that $v(x) \geq$ $\beta_{m}, \forall m \in \mathbb{N}$. Then

$$
F(x)>F(\hat{x})
$$


Otherwise, $F(x) \leq F(\hat{x})$. So that

$$
\beta_{m} \leq v(x) \leq \sup _{\substack{u \in X \\ F(u) \leq F(\hat{x})}} v(u)=0=v(\hat{x})<\beta_{m}
$$

which gives a contradiction. Then, property (16) implies that

$$
\inf \left(S_{\beta_{m}}\right)=\inf _{\substack{x \in X \\ v(x) \geq \beta_{m}}} F(x) \geq F(\hat{x})
$$

It follows from Proposition 4.2 that

$$
\lim _{m \rightarrow+\infty} \inf \left(S_{\beta_{m}}\right)=\inf (S) \geq F(\hat{x})
$$

So that $F(\hat{x})=\inf (S)$. Hence, $\hat{x}$ solves $(S)$.

The following theorem gives a necessary and sufficient optimality condition that reduces the problem $(S)$ to a min-max problem with compact convex linked constraints.

Theorem 4.4. Let Assumptions (3.1)-(3.6) and (4.1) hold. Let $\hat{x}$ be a feasible point of $(S)$. Then, $\hat{x}$ solves $(S)$ if and only if

i) Then, $v(\hat{x})=0$,

ii) inf $\underset{F(x) \leq F(\hat{x})}{x \in X} \sup _{\substack{y \in \mathbb{R}^{q} \\ g(x, y) \leq 0}} G(x, y)=0$.

Proof. Use Theorems 4.2 and 4.3.

For illustration, let us give the following simple example.

Example 4.1. Let $X=[-4,4], \quad F: \mathbb{R} \rightarrow \mathbb{R}, \quad G: \mathbb{R}^{2} \rightarrow \mathbb{R}, g=\left(g_{1}, g_{2}\right)$ : $\mathbb{R}^{2} \rightarrow \mathbb{R}^{2}$ be the functions defined by

$$
\left\{\begin{array} { l } 
{ F ( x ) = x ^ { 2 } + 1 } \\
{ G ( x , y ) = - 3 x ^ { 2 } - y ^ { 2 } + 1 }
\end{array} \quad \left\{\begin{array}{l}
g_{1}(x, y)=-x-y \\
g_{2}(x, y)=y-x
\end{array}\right.\right.
$$

For all $x \in[-4,4]$, we have $Y(x)=[-x, x] \subset[-4,4]$. Then, Assumptions (3.1) to (3.4) and (3.6) are satisfied. Moreover, we have

$$
\min _{x \in[-4,4]}\left(x^{2}+1\right)=1<\min _{\substack{x \in[-4,4] \\-3 x^{2}-y^{2}+1 \leq 0, \forall y \in[-x, x]}}\left(x^{2}+1\right)=\frac{4}{3} .
$$


Hence, Assumption (3.5) is satisfied. Let us verify Assumption (4.1). We have

$$
\begin{aligned}
& v(x)=3 x^{2}-1 \quad \partial v(x)=\left\{v^{\prime}(x)\right\}=\{6 x\} \\
& \{x \in[-4,4] / v(x)=0\}=\left\{-\frac{1}{\sqrt{3}}, \frac{1}{\sqrt{3}}\right\} \\
& \partial v\left(-\frac{1}{\sqrt{3}}\right)=\{-2 \sqrt{3}\} \quad \text { and } \partial v\left(\frac{1}{\sqrt{3}}\right)=\{2 \sqrt{3}\} .
\end{aligned}
$$

- For $x=\frac{1}{\sqrt{3}}$, let $x^{*}=2 \sqrt{3} \in \partial v\left(\frac{1}{\sqrt{3}}\right)$, and $\hat{x}=4 \in X$. Then

$$
x^{*}\left(\hat{x}-\frac{1}{\sqrt{3}}\right)>0 \text {. }
$$

- For $x=-\frac{1}{\sqrt{3}}$, let $x^{*}=-2 \sqrt{3} \in \partial v\left(-\frac{1}{\sqrt{3}}\right)$, and $\hat{x}=-4 \in X$. Then

$$
x^{*}\left(\hat{x}+\frac{1}{\sqrt{3}}\right)>0 \text {. }
$$

Hence, Assumption (4.1) is satisfied. Let us find the solutions of problem $(S)$ via the optimality conditions. For this, let us apply Theorem 4.4. Let $\bar{x}$ be a feasible point of $(S)$. Then, $\bar{x}$ solves $(S)$ if and only if $v(\bar{x})=0$ and

$$
\inf _{\substack{x \in X \\ F(x) \leq F(\bar{x})}} \sup _{\substack{y \in \mathbb{R} \\ g(x, y) \leq 0}} G(x, y)=0
$$

The equation $v(\bar{x})=0$, gives $\bar{x}=-\frac{1}{\sqrt{3}}$ and $\bar{x}=\frac{1}{\sqrt{3}}$. We have

$$
F\left(-\frac{1}{\sqrt{3}}\right)=F\left(\frac{1}{\sqrt{3}}\right)=\frac{4}{3} \text {. }
$$

Replacing the functions $F, G$, and $g$ by their expressions, we get

$$
\inf _{\substack{x \in X \\ F(x) \leq F(\bar{x})}} \sup _{\substack{y \in \mathbb{R} \\ g(x, y) \leq 0}} G(x, y)=\inf _{\substack{x \in[-4,4] \\ x^{2}+1 \leq \frac{4}{3}}} \sup _{y \in[-x, x]}\left(-3 x^{2}-y^{2}+1\right) .
$$

We have

$$
\sup _{y \in[-x, x]}\left(-3 x^{2}-y^{2}+1\right)=1-3 x^{2}
$$


and

$$
\inf _{\substack{x \in[-4,4] \\ x^{2}+1 \leq \frac{4}{3}}}\left(1-3 x^{2}\right)=0 .
$$

Therefore, $-\frac{1}{\sqrt{3}}$ and $\frac{1}{\sqrt{3}}$ are solutions of $(S)$.

\section{CONCLUSION}

Our investigation in this article concerned a class of nonsmooth generalized semi-infinite programming problems whose feasible sets are represented by a reverse convex constraint. So the use of reverse convex problems played an important role to establish our results. Then, for problem $(S)$, under appropriate assumptions, we have given necessary and sufficient optimality conditions. These optimality conditions are new and do not use differentiability assumptions. Normal cones, subdifferentials, and max-min problems are the main tools used to express these optimality conditions. In particular, using a new constraint qualification (Assumption (4.1)), we have provided a necessary and sufficient optimality condition which reduces $(S)$ to a min-max problem with compact convex linked constraints. Therefore, such an optimality condition provides an alternative to solve the considered class of generalized semi-infinite programming problems via min-max problems with linked constraints.

\section{REFERENCES}

1. R. Hettich and G. Still (1995). Second order optimality conditions for genaralized semi-infinite programming problems. Optimization 34:195-211.

2. H. Th. Jongen, J.-J. Rückman, and O. Stein (1998). Generalized semi-infinite optimization: A first-order optimality conditions and examples. Mathematical Programming 83:145-158.

3. N. Kanzi and S. Nobakhtian (2010). Necessary optimality conditions for nonsmooth generalized semi-infinite programming problems. European Journal of Operational Research 205:253-261.

4. N. Kanzi (2012). Convex generalized semi-infinite programming problems with constraint sets: Necessary conditions. Iranian Journal of Operations Research 3:24-32.

5. N. Kanzi (2013). Lagrange multiplier rules for non-differentiable DC geneneralized semi-infinite programming problems. Journal of Global Optimization 56:417-430.

6. J.-J. Rückmann and A. Shapiro (1999). First-order optimality conditions in generalized semiinfinite programming. Journal of Optimization Theory and Applications 101:677-691.

7. J.-J. Rückmann and A. Shapiro (2001). Second-order optimality conditions in generalized semiinfinite programming. Set-Valued Analysis 9:169-186.

8. O. Stein and G. Still (2000). On optimality conditions for generalized semi-infinite programming problems. Journal of Optimization Theory and Applications 104:443-458.

9. O. Stein (2001). First order optimality conditions for degenerate index sets in generalized semi-infinite programming. Mathematics of Operations Research 26:565-582.

10. G. Still (1999). Generalized semi-infinite programming : Theory and methods. European Journal of Operational Research 119:301-313.

11. J. J. Ye and S. Y. Wu (2008). First-order optimality conditions for generalized semi-infinite programming problems. Journal of Optimization Theory and Applications 137:419-434. 
12. Z. Chen and Z. Chen (2013). Optimality conditions for nonsmoth generalized semi-infinite programs. Abstract and Applied Analysis Article ID 131938, 1-8.

13. J.-B. Hiriart-Urruty and C. Lemarechal (1993). Convex Analysis and Minimization Algorithms, Vols. 1-2. Springer Verlag, New York.

14. R. T. Rockafellar (1970). Convex Analysis. Princeton University Press, Princeton, NJ.

15. J.-B. Hiriart-Urruty (1989). From convex optimization to nonconvex optimization. Part I: Necessary and sufficient conditions for global optimality. Nonsmooth Optimization and Related Topics (Erice 1988), 219-239. Ettore Majorana International Science Series: Physical Sciences, 43. Plenum, New York.

16. H. Tuy (1987). Convex Programs with additional reverse convex constraint. Journal of Optimization Theory and Applications 52:463-486.

17. H. Tuy and N. V. Thuong (1988). On the global minimization of a convex function under general nonconvex constraints. Applied Mathematics and Optimization 18:119-142.

18. A. Aboussoror (2009). Reverse convex programs: Stability and global optimality. Pacific Journal of Optimization 5:143-153. 